\title{
The Influence of Personas' Gender in Design
}

\author{
Milena Ribeiro Lopes* \\ Trinity College Dublin \\ Dublin, Ireland \\ lopesm@tcd.ie
}

\author{
Carl Vogel* \\ Trinity College Dublin \\ Dublin, Ireland \\ vogel@tcd.ie
}

\begin{abstract}
In mobile application design, the construction of personas is a wellknown technique that helps designers to empathise with users and to understand their needs. However, there are several issues that lead to stereotyped personas and men's needs being prioritised in design projects. The under-representation of women in design teams, insufficient user research, the assumption that the default user is a man, and the influence of personal beliefs in the decision making process all obstruct the inclusion of women's needs in the design process. Therefore, we propose the creation of gender-neutral personas that merge both the needs of men and women using two different approaches, and we compare them with traditional gendered personas. The results of the study confirm our hypothesis - the gender-neutral personas culminated in more gender-inclusive design requirements.
\end{abstract}

\section{CCS CONCEPTS}

- Human-centered computing $\rightarrow$ User centered design; • Social and professional topics $\rightarrow$ Gender.

\section{KEYWORDS}

gender-neutral persona, gender-inclusive design, mobile app development

\section{ACM Reference Format:}

Milena Ribeiro Lopes and Carl Vogel. 2021. The Influence of Personas' Gender in Design. In CHItaly 2021: 14th Biannual Conference of the Italian SIGCHI Chapter (CHItaly '21), July 11-13, 2021, Bolzano, Italy. ACM, New York, NY, USA, 8 pages. https://doi.org/10.1145/3464385.3464736

\section{INTRODUCTION}

Mobile application development is a field of knowledge in which concepts of design and HCI are merged to provide users with the best experience of technology. It is a discipline strongly influenced by user-centric approaches, which explains the use of specific tools such as persona, scenario and user journey. Technological development, however, risks being gender biased, for women are not well represented in design teams and in user research $[4,14,35]$, designers are likely to embed developments with their own opinions $[1,5,13,24-27]$, women have low influence in team's discussions

*Both authors contributed equally to this research.

This work is licensed under a Creative Commons

Attribution-NonCommercial-NoDerivs International 4.0 License.

CHItaly '21, July 11-13, 2021, Bolzano, Italy

(C) 2021 Copyright held by the owner/author(s)

ACM ISBN 978-1-4503-8977-8/21/06.

https://doi.org/10.1145/3464385.3464736
$[18,29,37]$, and because of a tendency to consider men as the "typical" users of technological artefacts [7, 15].

In order to counteract gender bias, we explore here the use of gender-neutral personas, in which men's and women's needs are merged into a single user description with no reference to gender. That is, the persona had no picture, no name, and the relevant information (age, city, income, study, job, hobbies, lifestyle, expectations, previous experience with apps, and so on) was neutralised through a gender-neutral textual description. Our study compared two approaches for gender-neutral personas with two gendered personas (a man and a woman). It was detected that the gender-neutral personas led to more inclusive results.

\section{RELATED WORK}

Different strategies have been suggested to counteract bias and gender issues in the design process, such as: participatory design [28], value sensitive design [15], reflective design [31], undoing design [9] and de-gendering design [5]. However, those strategies mostly provide guidelines to improve sensitivity and reflection on gender issues during the design process. There is still a lack of practical tools to mitigate the inscription of gender stereotypes in the user description and the tendency to prioritise men's needs.

In the design process, a persona should be guiding decisions at each stage in order to ensure that the development is in accordance with users' expectations. Nevertheless, personas can carry stereotypes of gender and/or stimulate a design team to stereotype users $[12,16,17,23,24,32,36]$. Several studies have been carried out to shed light on gender inclusivity, gender stereotypes and gender biases in the use of personas. A study that proposed the switch of personas' genders in order to bring awareness of stereotypes revealed positive results with regard to the inclusion of the needs of women and detection of stereotypes in projects [36]. During user studies proposed to investigate the effect of gender in the use of personas [10], participants proposed the use of a non-gendered persona (a persona with no declared gender), challenging the position that a persona should have a gender.

This idea of a gender-neutral persona was explored in the investigation of gender bias in design [22]. That study indicated that participants did not tend to perceive the gender-neutral persona more as a woman or as a man. They could engage with the user regardless of gender, providing evidence of the viability of using this approach. That study relied in the assumption that removing gender as an explicit quality of the description of personas would enable merging of different genders' needs into a single user description, and, accordingly, to mitigate bias towards one single gender group's needs. In that study, the creation of a gender-neutral persona was limited to the removal of gender traits, and no specific gendered user needs were added to that. However, removal of traits with gender associations is only one way to achieve neutrality; therefore, 
we explore an alternative. In this study, the concept of neutrality is extended to merge men's and women's traits and gender-neutral personas are compared to regular gendered personas to provide a deeper and better understanding of the application of the genderneutral approach.

Despite the fact that gender neutrality has been considered problematic in the design field, the use of the term "gender-neutral" in this research is justified for the connection of textual user descriptions with studies on gendered pronouns and neutral language. In the context of a design project, the term can imply a design that attempt to include all users by ignoring gender, and accordingly, gender issues that should be addressed in other to promote genderinclusivity in design $[11,30,34]$. Here, the term seems to fit better for textual description of intended users.

The concept of gender includes a comprehensive spectrum that includes transgender identities and nuances of gender expression, as explained by the concept of the "genderbread person" [19]. This research, however, is limited to two gender identities - man and woman - and does not explore the nuances between these two genders or the lack of both. Thus, we focus on these two poles of the spectrum in a more generic way rather than on different possible combinations of masculinities and femininities that can influence in design preferences. It is possible that the gender differences here are reduced to sex differences since nuances of gender are not explored; however, our interest is in preferences shaped by society and gender roles, and for this reason we did not ask the sex of participants, only their gender. It seemed beneficial to focus on the woman-man dynamic for it has a historical weight in the shaping of social interactions and gender roles, but this research is not at odds with a wider spectrum of gender identities and gender expression. Results obtained here provide a basis for exploring a more inclusive gender spectrum in the future.

\section{RESEARCH DESIGN}

The goal of this study is to determine whether gender-neutral personas promote the inclusion of men's and women's primary needs in a balanced way in the design process. In order to achieve this goal, an investigation of the impact of the neutral persona in the definition of requirements during the design of mobile applications was conducted through an online survey with professionals in creative fields. Data was gathered from 158 participants: 93 women and 65 men with age between 22 and 55 years old. Both age median and age mean was 35 years old.

Participants in this study were presented with two tasks in which they had to define priorities in the design of a fictitious online dating app based on the description of a persona. Each participant received one persona of the set that contained a woman, a man and two gender-neutral personas. One of the gender-neutral personas merged needs common to both men and women, and the other merged needs that were exclusive to each gender. In order to distinguish between the two gender-neutral personas, the one that depicts overlapping elements is referred to here as the common gender-neutral persona and the one that represents a mix of exclusively men's elements and exclusively women's elements is referred to as the mixed gender-neutral persona. Participants received those personas with no picture, no name, and a textual description in a neutral style. The persona that is a man is called Peter and the persona that is a woman is called Clara.

In the first task participants had to sort by the order of importance eight different "feelings" the persona could supposedly feel and to rate 12 features. Both feelings and features derived from the literature review on hookup and studies with users of online dating apps (e.g. [21]). The results were compared in order to understand whether the decisions made for gender-neutral personas met the needs of both gendered personas (man and woman).

The study was approved by the Research Ethics Committee of Trinity College Dublin. Participants were recruited by a call in social media groups for a snowball effect. Participants who volunteered did not receive any remuneration for participating. They were not told that gender was being analysed since that could influence their decisions, but they were given the option to receive a debriefing email with more information and results.

\subsection{Construction of personas: selection of masculine and feminine traits}

The creation of the personas relied on studies on users' experiences of dating applications. Research on hookup culture and user studies with online dating users brought to light gender dynamics that revealed women's and men's preferences, expectations and perceptions of the service. In previous studies, for example, it was detected that men were much more likely to lose respect for women who hookup than for men $[2,6]$, women were likely to have their pleasure neglected during such encounters [2, 3], those who benefited from hookups were mostly men [33], women (users of dating apps) were less likely to report to be looking for sex and more likely to feel disrespected [20], and women would prefer to focus on relationships [8] rather than hookups.

The information collected in a study of gender differences on dating applications [21] guided the creation of the set of personas. That study revealed that men were more likely than women to benefit from "easy dates setting" and to have a respectful experience, while women were more likely to perceive the application as "too sexual" and "superficial", that both genders felt confident using the application, and both benefited from getting to know new people through the application. Both men and women found it boring for its lack of dynamism and untrustworthy for people often lying about themselves. Women and men shared openness to different sorts of interaction (friendship, casual dates, longterm relationships) and the same perception that men are mainly looking for sex while women are looking for a relationship. Both expressed belief that their experience would have been better if the matching system was more accurate and sensitive to their preferences, since they felt the system was somehow inefficient. Additionally, in order to address some perceptions of men's and women's experiences of dating apps that were not detected through prior statistical tests, it was necessary to select their most frequent answers to create the set of personas. With this, it was possible to determine which answers were more common to men, to women and to both (see Table 1).

\subsection{Method of analysis}

To verify whether gender-neutral personas promote the inclusion of men's and women's needs in a balanced way in a design project, 
Table 1: Selection of elements that describe women's and men's experience of dating apps."Motivations" refers to the reasons for installing the app; "1st impressions" refers to initial reaction to the application; "1st matches" refers to initial responses to first connections; "match system" refers to the perception of the interaction design; "benefit of use" refers to positive experiences; "downside" refers to negative experiences; "felt respected" refers to whether users felt respected during their experience; "uninstalled" refers to reasons for removing the app, for those who had.

\begin{tabular}{|c|c|c|c|}
\hline \multirow[b]{2}{*}{ Question } & \multicolumn{3}{|c|}{ Gendered categories of answer } \\
\hline & Women & Man & Both \\
\hline Motivations & & & $\begin{array}{l}\text { hookup } \\
\text { friendship } \\
\text { relationship }\end{array}$ \\
\hline 1st impression & superficial & & great \\
\hline 1st matches & & & confident \\
\hline Match system & effective & superficial & $\begin{array}{r}\text { inefficient } \\
\text { OK }\end{array}$ \\
\hline Benefit of use & romance & easy dates & friends \\
\hline Downside & $\begin{array}{r}\text { offences } \\
\text { too sexual } \\
\text { people lie }\end{array}$ & $\begin{array}{r}\text { boring } \\
\text { bad situations }\end{array}$ & \\
\hline Felt respected & no/not fully & & yes \\
\hline Uninstalled & bad experience & & relationship \\
\hline
\end{tabular}

it was necessary to compare the prioritisation of design elements of the four personas. The Kendall's tau correlation was used to compare the elements' ordering, and the Wilcoxon test was used to detect significant differences between each individual design element. In order to identify the most important feelings and features for each persona, the elements within the ranks were organised in three categories: high, medium and low priorities (see Figures 1 and 2). For task 1, medians between 1 and 3 (inclusive) were defined as high priority, medians between 4 and 5 (inclusive) as medium priority, and medians higher than 5 as low priority. For task 2, medians higher than 4 were defined as high priority, medians between 3 and 4 (inclusive) as medium priority, and medians lower than 3 as low priority.

The ranks in task 1 were organised from 1 to 8 with the greatest value given to the element at the top, and the ranks in task 2 in a reverse rationale, from 5 to 1 , where the value of 5 represents the greatest value in the rating scale. The data ranks were sorted by medians, but elements with identical medians were subsequently sorted by means. For a comparison between participants' gender, ranks were also created with the scores for men and women participating in the studies for each task and persona (see Figures 1 and 2). Thus, different ranks were generated according to the variable being analysed: participants' gender and persona's gender.

\section{RESULTS}

\subsection{Test 1: Differences between personas}

By analysing the similarities using Kendall's tau correlations, it was possible to understand that the differences in the order of elements within the ranks produced by participants in the study for each persona are not significant, in general. The resulting rank order for task 1 and task 2 are displayed in Figure 1 and 2, respectively. A comparison between ranks for task 1 revealed that Peter's and Clara's ranks are more similar to the common neutral persona's rank than to the mixed neutral persona's rank. The strongest correlations for task 1, however, are between Clara's and Peter's ranks and between Clara's and the common neutral personas' ranks $(\tau=$ 0.929 and $p<0.01)$. The least significant correlations are between Peter's and the mixed neutral personas' ranks and between the two gender-neutral personas ( $\tau=0.643$ and $p<0.05$ ). With regard to task 2, Peter's rank is more correlated to the common persona's rank $(\tau=0.848$ and $p<0.01)$ while Clara's rank is more correlated to the mixed persona's rank $(\tau=0.970$ and $p<0.01)$. The strongest correlation is between Clara's and the mixed persona's ranks. The least significant correlation is between Peter's and the mixed persona's ranks ( $\tau=0.788$ and $p<0.01)$.

\begin{tabular}{|c|c|c|c|c|c|}
\hline \multicolumn{3}{|c|}{ Persona: Peter } & \multicolumn{3}{|c|}{ Persona: Clara } \\
\hline Feelings & $M$ & $\mu$ & Feelings & $M$ & $\mu$ \\
\hline Safe & 2 & 2.93 & Safe & 2 & 3.16 \\
\hline Respected & 4 & 3.67 & Respected & 3 & 3.33 \\
\hline Comfortable & 4 & 3.93 & Comfortable & 3 & 3.72 \\
\hline Happy & 4 & 4.09 & Confident & 5 & 4.33 \\
\hline Confident & 4 & 4.44 & Happy & 5 & 4.44 \\
\hline Appreciated & 5 & 4.41 & Appreciated & 6 & 5.08 \\
\hline In control & 6 & 5.41 & In control & 6 & 5.08 \\
\hline Engaged & 8 & 7.09 & Engaged & 8 & 6.83 \\
\hline \multicolumn{3}{|c|}{ Persona: Mixed } & \multicolumn{3}{|c|}{ Persona: Common } \\
\hline Feelings & M & $\mu$ & Feelings & $M$ & $\mu$ \\
\hline Respected & 2 & 2.85 & Safe & 2 & 2.92 \\
\hline Safe & 2 & 2.87 & Comfortable & 3 & 3.23 \\
\hline Comfortable & 4 & 3.90 & Respected & 3 & 3.68 \\
\hline Appreciated & 4 & 4.56 & Confident & 4.5 & 4.50 \\
\hline Confident & 5 & 4.68 & Happy & 5 & 4.65 \\
\hline In control & 5 & 4.87 & Appreciated & 5 & 4.76 \\
\hline Happy & 5 & 4.90 & In control & 5 & 5.10 \\
\hline Engaged & 8 & 7.31 & Engaged & 8 & 7.07 \\
\hline
\end{tabular}

Figure 1: Ranks for Task 1: data ordered by medians and means values.

The correlations between high priorities are indicated in the diagram in the Figure 3. Considering results for task 1 and 2 together, the mixed persona presents six high priorities, the common persona five, Peter two and Clara seven. In task 1, the mixed persona presents the single feeling rated as high for Peter and two of the three feelings 


\begin{tabular}{|c|c|c|c|c|c|}
\hline \multicolumn{3}{|c|}{ Persona: Peter } & \multicolumn{3}{|c|}{ Persona: Clara } \\
\hline Features & $M$ & $\mu$ & Features & $M$ & $\mu$ \\
\hline Common interests & 5 & 4.54 & Relationship mode & 5 & 4.47 \\
\hline Join a group & 4 & 4.06 & Stay safe & 5 & 4.03 \\
\hline Relationship mode & 4 & 3.90 & Common interests & 5 & 4.25 \\
\hline Stay safe & 4 & 3.81 & Rate users & 4.5 & 4.13 \\
\hline Rate Users & 4 & 3.79 & Join a group & 4 & 3.94 \\
\hline Chemistry & 4 & 3.76 & Chemistry & 4 & 3.72 \\
\hline Self awareness & 3 & 3.23 & Self awareness & 4 & 3.50 \\
\hline Smart move & 3 & 2.95 & Match boost & 3 & 2.86 \\
\hline Match boost & 3 & 2.93 & Smart move & 3 & 2.75 \\
\hline Flirting tips & 3 & 2.47 & Flirting tips & 3 & 2.69 \\
\hline Time is ticking & 2 & 2.71 & Time is ticking & 2.5 & 2.55 \\
\hline Gift voucher & 2 & 2.04 & Gift voucher & 2 & 2.38 \\
\hline \multicolumn{3}{|c|}{ Persona: Mixed } & \multicolumn{3}{|c|}{ Persona: Common } \\
\hline Features & $M$ & $\mu$ & Features & $M$ & $\mu$ \\
\hline Relationship mode & 5 & $4.5 I$ & Common interests & 5 & 4.44 \\
\hline Stay safe & 5 & 4.46 & Stay safe & 4.5 & 3.78 \\
\hline Common interests & 5 & 4.41 & Relationship mode & 4 & 3.95 \\
\hline Rate users & 5 & 4. 17 & Join a group & 4 & 3.94 \\
\hline Join a group & 4 & 3.95 & Chemistry & 4 & 3.91 \\
\hline Chemistry & 4 & 3.70 & Rate users & 4 & 3.65 \\
\hline Self awareness & 3 & 3.17 & Self awareness & 3 & 3.21 \\
\hline Match boost & 3 & 2.80 & Match boost & 3 & 2.86 \\
\hline Flirting tips & 3 & 2.75 & Smart move & 3 & 2.84 \\
\hline Smart move & 3 & 2.73 & Flirting tips & 2 & 2.42 \\
\hline Time is ticking & 3 & 2.67 & Time is ticking & 2 & 2.08 \\
\hline Gift voucher & 2 & 2.35 & Gift voucher & I & 2.02 \\
\hline
\end{tabular}

$\operatorname{median}(\mathrm{M})>4$

median (M) 3-4

median $(M)<3$

Figure 2: Ranks for Task 2: data ordered by medians and means values.

rated high for Clara. The common persona, in turn, presents the single feeling rated as high for Peter and all three feelings rated as high for Clara. Thus, the mixed one encompasses two of the three feelings rated high among the two groups and the common persona encompasses three of the three feelings rated high. In task 2 , the mixed persona presents the single feature rated high for Peter and all the four features rated high for Clara. The common persona, in turn, presents the feature rated high for Peter and two of the four features rated high for Clara. Hence, the mixed persona encompasses all four features rated high among the two groups while the common persona encompasses two. Peter's high priorities coincide with both mixed and common personas for the two tasks.

When analysing the two tasks together, it was also possible to detect that the mixed gender-neutral persona met six of the Clara's and Peter's top priorities while the common gender-neutral persona met five of those priorities. Designing for Peter would lead to the exclusion of five of Clara's priorities for task 1 and 2. Designing for Clara's persona would ensure that both users needs were met because the Peter's priorities are also included in Clara's priorities. However, in this study, Peter engendered only two priorities while Clara generated seven priorities, among feelings and features. For this reason it is not safe to conclude that this case applies to every

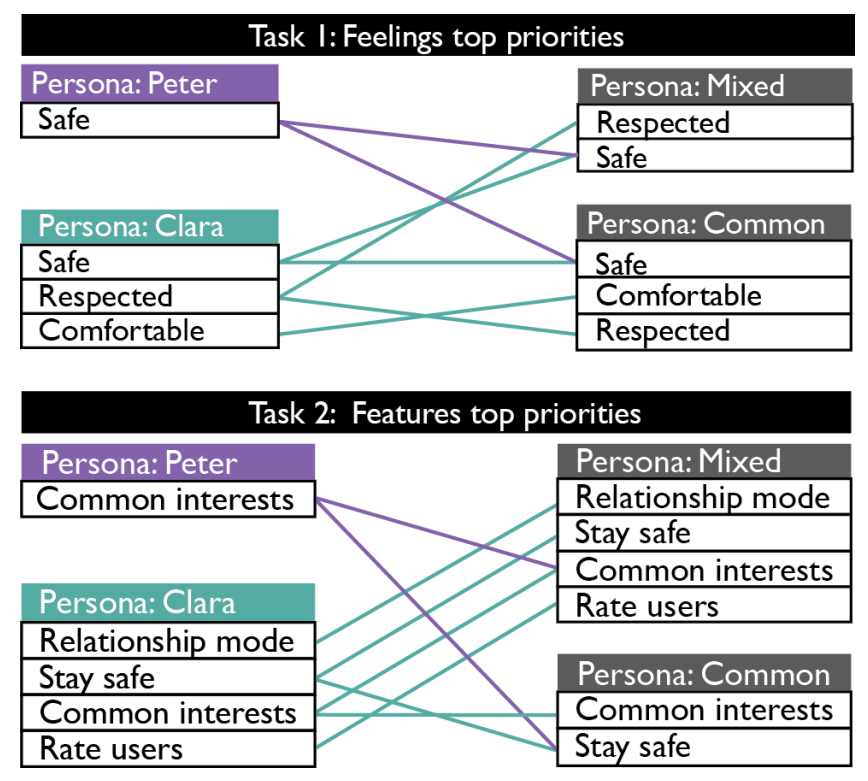

Figure 3: Correlation between personas' top rated elements of task 1 and 2 .

other case. Merging the needs of both men and women (or other gender groups) would probably be ideal to ensure that both groups' needs are met. Thus, using a gender-neutral persona should return more inclusive design requirements since the focus is given to the users' needs and designers would be less likely to empathise more with one gender than other. That is, considering the ideal scenario in which those priorities assigned by participants for each persona matched the real needs of men and women. However, it still remains unclear which persona would serve better for a gender-inclusive approach that takes into account women's and men's needs in a balanced way. So far, two concepts of neutrality were used in the creation of the personas, but a persona that merges both the needs in common and those exclusive of each gender should also be considered and tested in the future. In this study, both genderneutral personas provided more inclusive priorities but the mixed gender-neutral one would met more of both Peter's and Clara's priorities.

The analysis of individual elements within the ranks (see Table 2) through Wilcoxon tests revealed that, between Peter and Clara, there was a significant difference for the "relationship" feature, which was rated higher for the Clara persona. Between the Peter persona and the mixed gender-neutral one, there were significant differences with regard to feeling "respected" and to the "relationship" and the "stay safe" features. All the three elements were considered more important for the mixed persona. Between Clara and the common gender-neutral personas, there is a significant difference for the "relationship" feature, which was preferred for Clara. Between the common and the mixed gender-neutral personas, there is a significant difference for the "relationship" feature, which was preferred for the mixed gender-neutral persona, and differences approaching significance for feeling "respected" and the "stay safe" feature. No significant differences were found by comparing Peter 
and the common gender-neutral personas, or by comparing Clara and the mixed gender-neutral personas. By analysing the distributions for the "respected" feeling, and for the "relationship mode" and "stay safe" features, it became clearer that the common persona is more similar to Peter and that the mixed persona represents better Clara's qualities. Those statistically significant differences revealed that priorities derived from problematic issues in the user experience of dating apps would be much more likely to be addressed in design by using the mixed persona than by using the common persona.

Table 2: Differences in priority setting for personas in task 1 and 2, according to personas' gender. Elements of Task 1 refer to "feelings" and elements of Task 2 refer to "features". Statistical significance in Wilcoxon test is indicated with asterisks $(*-p<0.05 ; * *-p<0.01 ; * * *-p<0.001)$.

\begin{tabular}{llllr}
\hline Task & Personas' Pair & Element & P-value & Prioritised for \\
\hline Task 1 & Peter X Mixed & Respected & $0.03^{*}$ & Mixed \\
Task 1 & Common X Mixed & Respected & 0.050 & Mixed \\
\hline Task 2 & Clara X Peter & Relationship & $0.024^{*}$ & Clara \\
Task 2 & Peter X Mixed & Relationship & $0.009^{* *}$ & Mixed \\
Task 2 & Peter X Mixed & StaySafe & $0.007^{* *}$ & Mixed \\
Task 2 & Clara X Common & Relationship & $0.042^{*}$ & Clara \\
Task 2 & Common X Mixed & Relationship & $0.019^{*}$ & Mixed \\
Task 2 & Common X Mixed & StaySafe & 0.052 & Mixed
\end{tabular}

\subsection{Test 2: Differences between personas by participants' gender}

The rank orders for task 1 and task 2 according to participants' gender are displayed in Figures 4 and 5, respectively. Kendall's tau correlation test shows that Clara's and Peter's ranks are statistically similar for each persona in task 1 and 2, except for women's and men's judgments for the common neutral persona in task 1 which ranks do not reveal similarities $(\tau=0.429$ and $p=0.138)$. For the common persona in task 1 , however, some major differences appear. For example, the feeling of "respect" drops from the 3rd position in the rank produced by women to the 7th position in men's ranks, and feeling "in control" rises from the 7th position in women's rank to the 4th position in the men's one (see Figure 4). Furthermore, despite the statistical similarity between the ranks produced by women and men for the common persona in task 2 , a large difference in positions appear for some elements, such as the "chemistry" feature that rises from 5th position in women's rank to the 1st in the men's one or the "match boost" that drops from 7th in women's rank to the 11th in the men's one (see Figure 5). The differences that emerged in the ranks produced by men and women for the common gender-neutral persona indicate that participants can have different perceptions of users' needs and that the absence of gender can make more room for the influence of personal opinions. In order to understand those effects, it would be necessary to compare the use of two gendered personas with the use of a single gender-neutral persona in the future.

Some differences emerged by analysing the top priorities assigned by men and women for each of the four personas. In task 1 ,

\begin{tabular}{|c|c|c|c|c|c|}
\hline \multicolumn{6}{|c|}{ Male Persona (Peter) - Task I } \\
\hline \multicolumn{3}{|c|}{ Women TI } & \multicolumn{3}{|c|}{ Men TI } \\
\hline Feelings & $M$ & $\mu$ & Feelings & $M$ & $\mu$ \\
\hline Safe & 2 & 2.69 & Safe & 2 & 3.29 \\
\hline Respected & 4 & 3.53 & Happy & 3 & 3.29 \\
\hline Comfortable & 4 & 3.88 & Comfortable & 3 & 4.00 \\
\hline Confident & 4 & 4.26 & Respected & 4 & 3.88 \\
\hline Happy & 4.5 & 4.61 & Appreciated & 4 & 4.11 \\
\hline Appreciated & 5 & 4.61 & Confident & 5 & 4.70 \\
\hline In control & 6 & 5.53 & In control & 6 & 5.23 \\
\hline Engaged & 8 & 6.84 & Engaged & 8 & 7.47 \\
\hline \multicolumn{6}{|c|}{ Female Persona (Clara) - Task I } \\
\hline \multicolumn{3}{|c|}{ Women TI } & \multicolumn{3}{|c|}{ Men TI } \\
\hline Feelings & $\bar{M}$ & $\mu$ & Feelings & $M$ & $\mu$ \\
\hline Safe & 2.5 & 3.3 & Safe & 2 & 3.00 \\
\hline Respected & 3 & 3.25 & Comfortable & 3 & 3.75 \\
\hline Comfortable & 3 & 3.70 & Respected & 3.5 & 3.43 \\
\hline Confident & 5 & 4.30 & Confident & 4.5 & 4.37 \\
\hline Happy & 5 & 4.45 & Happy & 5 & 4.43 \\
\hline In control & 5.5 & 4.85 & Appreciated & 6 & 4.87 \\
\hline Appreciated & 5.5 & 5.25 & In control & 6 & 5.37 \\
\hline Engaged & 8 & 6.90 & Engaged & 7.5 & 6.75 \\
\hline \multicolumn{6}{|c|}{ Mixed Persona (Peter) - Task I } \\
\hline \multicolumn{3}{|c|}{ Women TI } & \multicolumn{3}{|c|}{ Men TI } \\
\hline Feelings & $M$ & $\mu$ & Feelings & $M$ & $\bar{\mu}$ \\
\hline Respected & 2 & 2.72 & Respected & 2 & 3.06 \\
\hline Safe & 2 & 2.80 & Safe & 3 & 3.00 \\
\hline Comfortable & 4 & 4.16 & Comfortable & 3.5 & 3.50 \\
\hline Appreciated & 4 & 4.60 & Appreciated & 4.5 & 4.50 \\
\hline Confident & 5 & 4.52 & Happy & 5 & 4.43 \\
\hline In control & 5 & 4.64 & Confident & 5 & 4.93 \\
\hline Happy & 6 & 5.20 & In control & 5.5 & 5.25 \\
\hline Engaged & 8 & 7.36 & Engaged & 8 & 7.25 \\
\hline \multicolumn{6}{|c|}{ Common Persona (Clara) - Task I } \\
\hline \multicolumn{3}{|c|}{ Women TI } & \multicolumn{3}{|c|}{ Men TI } \\
\hline Feelings & $\bar{M}$ & $\mu$ & Feelings & $M$ & $\mu$ \\
\hline & 2 & 2.68 & Safe & 2.5 & 3.25 \\
\hline Comfortable & 2.5 & 3.09 & Comfortable & 3 & 3.43 \\
\hline Respected & 3 & 2.68 & Confident & 4 & 3.87 \\
\hline Happy & 4.5 & 4.59 & In control & 4.5 & 4.31 \\
\hline Confident & 5 & 4.95 & Appreciated & 5 & 4.00 \\
\hline Appreciated & 6 & 5.31 & Happy & 5 & 4.75 \\
\hline In control & 6 & 5.68 & Respected & 5.5 & 5.06 \\
\hline Engaged & 8 & 6.95 & Engaged & 8 & 7.25 \\
\hline
\end{tabular}

$\operatorname{median}(\mathrm{M}) \leq 3$

median $(M)>3$

median $(M)>5$

Figure 4: Ranks of women and men for task 1: data ordered by medians and means values.

men produced ranks with more priorities than women when designing for Peter, women produced ranks with more priorities than men when designing for Clara and the common gender-neutral personas. No gender differences in the number of priorities were detected for the mixed gender-neutral persona. In task 2, women assigned more priorities to Peter, Clara and the mixed gender-neutral personas' 
ranks. Only for the common gender-neutral persona is it that men assigned more priorities than women.

Considering that the rank produced for the common genderneutral persona is very similar to the rank produced for Clara in task 1 and considering that women tended to assign more priorities than men to Clara and the common gender-neutral persona and men to Peter, it was possible to infer that participants were more likely to agree with each other when designing for personas of their own gender, or perceived as such, with regard to the users' "feelings" (task 1). This effect might also indicate that designers were more likely to understand better the needs of personas of their own gender - perhaps this is the reason why the agreement between participants for important design elements was greater for those personas. In task 2, in turn, which is related to the application's features, such an effect cannot be perceived since women assign more priorities to Peter than men do. Women, in comparison to men, assigned more priorities to Peter, Clara and the mixed genderneutral persona while men assigned more priorities to the common gender-neutral persona. Recall from task 2 that the rank produced for the mixed gender-neutral persona was more similar to the rank produced for Clara than for Peter, and the rank produced for the common gender-neutral was equally similar to Peter's and Clara's ranks. Women tended to assign more priorities than men for the ranks in task 2 (14 priorities assigned by women, 10 assigned by men) but there were no differences in the sum of priorities in task 1 (9 priorities each). Thus, regarding features, it is possible that women perceive more users' needs than men do, in general, but more study is required to investigate whether those perceived needs suit the reality of users' needs.

In the analysis of high priorities in the ranks produced by men and women participating in the study, it became visible that both men and women were more likely to assign high priority to feeling "safe" for every persona, but men were more likely than women to prioritise feeling "happy" and "comfortable" when deciding for Peter, while women were more likely than men to prioritise feeling "respected" and "comfortable" when designing for Clara. Women were also more likely to prioritise the feeling of "respect" for the common persona than men.

Comparison between the distribution of values between men and women participating in the study was conducted using Wilcoxon tests (see Table 3). In task 1, it was revealed that men were more likely than women to prioritise the "happy" feeling for Peter and the "appreciated" and "in control" feelings for common neutral persona, while women were more likely to prioritise the "respected" feeling for the common neutral persona, in comparison to men. In task 2, women were more likely than men to prioritise the "stay safe" and "time is ticking" features when designing for Clara and the "match boost" feature when designing for the common neutral persona. Men, in turn, were more likely than women to prioritise the "chemistry" feature when designing for the common neutral personas, in comparison to women.

\section{CONTRIBUTIONS, CHALLENGES, AND CONSTRAINTS}

In this study the use of gender-neutral personas resulted design priorities that merge both men's and women's priorities. The idea of
Table 3: Differences in priority setting for each persona in task 1 and 2, according to participants' gender. Elements of Task 1 refer to "feelings" and elements of Task 2 refer to "features". Statistical significance in Wilcoxon test is indicated with asterisks $(*-p<0.05 ; * *-p<0.01 ; * * *-p<0.001)$.

\begin{tabular}{llllr}
\hline Task & Persona & Element & P-value & Prioritised by \\
\hline Task 1 & Common & Appreciated & $0.045^{*}$ & Men \\
Task 1 & Common & In control & $0.017^{*}$ & Men \\
Task 1 & Common & Respected & $0.001^{* *}$ & Women \\
Task 1 & Peter & Happy & $0.039^{*}$ & Men \\
\hline Task 2 & Clara & Stay Safe & $0.019^{*}$ & Women \\
Task 2 & Clara & Time is Ticking & $0.017^{*}$ & Women \\
Task 2 & Common & Chemistry & $0.010^{*}$ & Men \\
Task 2 & Common & Match Boost & $0.047^{*}$ & Women
\end{tabular}

using gender-neutral personas in design may have some constraints, which can be anticipated here. The use of a gender-neutral persona may not prevent one from unconsciously assigning a gender to the persona, but as said before, it would help to retain focus on the persona's needs rather than reinforcing stereotypes and gender scripts. Some projects would need a set with more than one genderneutral persona to attend to important groups of stakeholders, which include users, although it is suggested to keep personas narrow and focused [13], which it is not always possible. In that case, a suggestion is to approach gender neutrality in each group, if they are composed by women and men (users). Otherwise, there might be no need to use a gender-neutral persona. Another scenario in which the gendered approach would probably remain the best option is when the project targets a specific gender. Benefits of using neutral personas in gendered scenarios can be explored in future research. Future discussions on the approach to gender neutrality and the technique proposed here can help to improve design tools and the design process as a whole since gender comes to light as a very important factor to take into account in mobile application development and other areas of design that can benefit from an inclusive perspective.

The use of gender-neutral personas in this study showed that depending on the task assigned to participants, one gender-neutral persona can be more inclusive of women's and men's needs than the other. However, for each task, at least one gender-neutral persona was capable of encompassing both men's and women's top priorities for that task. It remains to investigate whether the priorities assigned to those users match the reality. Furthermore, the study is limited to two approaches for creating gender-neutral personas, one that merges traits and needs in common between men and women and the other that merges needs that are exclusive to each gender. A strategy to deal with conflicting priorities between genders would be required, since needs of one group will eventually oppose the other. It remains to explore the effects of using a gender-neutral persona that merges what is in common and what is exclusive, which would probably motivate more inclusivity since all the needs are combined into a single persona. However, the idea of this first study on gender-neutral personas was exactly to 


\begin{tabular}{|c|c|c|c|c|c|}
\hline \multicolumn{6}{|c|}{ Male Persona (Peter) - Task 2} \\
\hline \multicolumn{3}{|c|}{ Women T2 } & \multicolumn{3}{|c|}{ Men T2 } \\
\hline Features & $M$ & $\mu$ & Features & $M$ & $\mu$ \\
\hline Common interests & 5 & 4.60 & Common interests & 5 & 4.47 \\
\hline Join a group & 5 & 4.15 & Join a group & 4 & 3.94 \\
\hline Chemistry & 5 & 3.84 & Relationship mode & 4 & 3.94 \\
\hline Relationship mode & 4 & 3.88 & Rate users & 4 & 3.94 \\
\hline Stay safe & 4 & 3.76 & Stay safe & 4 & 3.88 \\
\hline Rate Users & 4 & 3.69 & Chemistry & 4 & 3.64 \\
\hline Self awareness & 3.5 & 3.26 & Self awareness & 3 & 3.17 \\
\hline Match boost & 3 & 3.26 & Smart move & 3 & 2.93 \\
\hline Smart move & 3 & 2.96 & Flirting tips & 3 & 2.62 \\
\hline Flirting tips & 2.5 & 2.38 & Gift voucher & 2.5 & 2.62 \\
\hline Time is ticking & 2 & 2.84 & Time is ticking & 2 & 2.50 \\
\hline Gift voucher & 2 & 2.26 & Match boost & 2 & 2.41 \\
\hline
\end{tabular}

\begin{tabular}{|c|c|c|c|c|c|}
\hline \multicolumn{6}{|c|}{ Female Persona (Clara) - Task 2} \\
\hline \multicolumn{3}{|c|}{ Women T2 } & \multicolumn{3}{|c|}{ Men T2 } \\
\hline Features & $M$ & $\mu$ & Features & M & $\mu$ \\
\hline Stay safe & 5 & 4.60 & Relationship mode & 5 & 4.56 \\
\hline Relationship mode & 5 & 4.40 & Common interests & 4.5 & 4.18 \\
\hline Common interests & 5 & 4.30 & Rate users & 4 & 4.12 \\
\hline Rate Users & 5 & 4.15 & Stay safe & 4 & 3.93 \\
\hline Join a group & 4 & 4.10 & Join a group & 4 & 3.75 \\
\hline Chemistry & 4 & 3.95 & Chemistry & 3 & 3.43 \\
\hline Self awareness & 3 & 3.80 & Self awareness & 3 & 3.12 \\
\hline Time is ticking & 3 & 3.00 & Match boost & 2.5 & 2.87 \\
\hline Smart move & 3 & 2.90 & Smart move & 2.5 & 2.56 \\
\hline Match boost & 3 & 2.85 & Flirting tips & 2.5 & 2.50 \\
\hline Flirting tips & 3 & 2.85 & Gift voucher & 2 & 2.25 \\
\hline Gift voucher & 2.5 & 2.50 & Time is ticking & 2 & 2.00 \\
\hline
\end{tabular}

\begin{tabular}{|c|c|c|c|c|c|}
\hline \multicolumn{6}{|c|}{ Mixed Persona - Task 2} \\
\hline \multicolumn{3}{|c|}{ Women T2 } & \multicolumn{3}{|c|}{ Men T2 } \\
\hline Features & $M$ & $\mu$ & Features & $M$ & $\mu$ \\
\hline Relationship mode & 5 & 4.56 & Relationship mode & 5 & 4.43 \\
\hline Common inte & 5 & 4.52 & Common inte & 5 & 4.25 \\
\hline Stay safe & 5 & 4.52 & Stay safe & 4.5 & 4.37 \\
\hline Rate Users & 5 & 4.24 & Chemistry & 4.5 & 3.87 \\
\hline Join a & 5 & 4.12 & Rate users & 4 & 4.06 \\
\hline Chemistry & 4 & 3.60 & Join a group & 3.5 & 3.68 \\
\hline Self awareness & 4 & 3.44 & Match boost & 3 & 2.93 \\
\hline Flirting tips & 3 & 2.83 & Smart move & 2.5 & 2.62 \\
\hline Smart move & 3 & 2.80 & Flirting tips & 2.5 & 2.62 \\
\hline Match boost & 3 & 2.72 & Self awareness & 2 & 2.75 \\
\hline Time is ticking & 3 & 2.72 & Time is ticking & 2 & 2.60 \\
\hline Gift voucher & 2 & 2.45 & Gift voucher & 2 & 2.18 \\
\hline
\end{tabular}

\begin{tabular}{|c|c|c|c|c|c|}
\hline \multicolumn{6}{|c|}{ Common Persona - Task 2} \\
\hline \multicolumn{3}{|c|}{ Women T2 } & \multicolumn{3}{|c|}{ Men T2 } \\
\hline Features & $M$ & $\mu$ & Features & $M$ & $\mu$ \\
\hline Common interests & 5 & 4.59 & Chemistry & 5 & 4.43 \\
\hline Stay safe & 5 & 3.81 & Common interests & 5 & 4.25 \\
\hline Join a group & 4 & 4.00 & Rate users & 4.5 & 4.00 \\
\hline Relationship mode & 4 & 3.95 & Relationship mode & 4 & 3.93 \\
\hline Chemistry & 4 & 3.52 & Join a group & 4 & 3.87 \\
\hline Rate Users & 3.5 & 3.40 & Stay safe & 4 & 3.75 \\
\hline Match boost & 3 & 3.27 & Self awareness & 3.5 & 3.43 \\
\hline Self awareness & 3 & 3.04 & Smart move & 3 & 3.06 \\
\hline Smart move & 2.5 & 2.68 & Flirting tips & 2.5 & 2.56 \\
\hline Flirting tips & 2 & 2.31 & Gift voucher & 2 & 2.37 \\
\hline Time is ticking & 2 & 2.19 & Match boost & 2 & 2.31 \\
\hline Gift voucher & I & 1.77 & Time is ticking & 2 & 1.93 \\
\hline
\end{tabular}

$\operatorname{median}(M)>4$

median (M) 3-4

$\operatorname{median}(\mathrm{M})<3$ explore those two approaches separately in order to understand how it leads to differences in the decision making process. From a statistical point of view, the results disclosed here are constrained by the small sample size. More statistical differences could possibly emerge with a bigger sample.

\section{ACKNOWLEDGMENTS}

The first author would like to acknowledge the CAPES foundation for funding this research. This research is also supported by Science Foundation Ireland (SFI) through the CNGL Programme (Grant 12/CE/I2267 and 13/RC/2106) in the ADAPT Centre (www.adaptcentre.ie).

\section{REFERENCES}

[1] Madeleine Akrich. 1995. User representations: Practices, methods and sociology. In Managing technology in society: the approach of constructive technology assessment, Arie Rip, Thomas J Misa, and Johan Schot (Eds.). Pinter Publish, London, UK, 167-184.

[2] Rachel Allison and Barbara Risman. 2013. A double standard for "Hooking Up": How far have we come toward gender equality? Social Science Research 42, 5 (2013), 1191-1206.

[3] Elizabeth A. Armstrong, Paula England, and Alison C. K. Fogarty. 2012. Accounting for Women's Orgasm and Sexual Enjoyment in College Hookups and Relationships. American Sociological Review 77, 3 (2012), 435-462.

[4] Catherine Ashcraft, Brad McLain, and Elizabeth Eger. 2016. Women in tech: The facts. National Center for Women \& Technology (NCWIT), Boulder, CO, USA.

[5] Corinna Bath. 2014. Searching for methodology. In Gender in Science and Technology. Transcript Verlag, Bielefeld, DE, 57-78.

[6] Kathleen A. Bogle. 2008. Hooking Up: Sex, Dating, and Relationships on Campus. NYU Press, New York, NY, USA.

[7] Adam Bradley, Cayley MacArthur, Mark Hancock, and Sheelagh Carpendale. 2015. Gendered or neutral? considering the language of HCI. In Proceedings of Graphics Interface 2015 (GI 2015), Hao Zhang and Tony Tang (Eds.). Canadian Information Processing Society, Toronto, Ontario, Canada, 163-170.

[8] Carolyn Bradshaw, Arnold S Kahn, and Bryan K Saville. 2010. To hook up or date: Which gender benefits? Sex Roles 62, 9-10 (2010), 661-669.

[9] Martin Brigham and Lucas D Introna. 2007. Invoking politics and ethics in the design of information technology: undesigning the design. Ethics and Information Technology 9, 1 (2007), 1-10.

[10] Margaret Burnett, Anicia Peters, Charles Hill, and Noha Elarief. 2016. Finding gender-inclusiveness software issues with GenderMag: a field investigation. In Proceedings of the 2016 CHI Conference on Human Factors in Computing Systems (CHI'16). ACM, New York, NY, USA, 2586-2598.

[11] Elizabeth F. Churchill. 2010. Sugared Puppy-dog Tails: Gender and Design. Interactions 17, 2 (2010), 52-56.

[12] Allan Cooper. 1999. The inmates are running the asylum. Sams Publishing, Indianapolis, IN, USA.

[13] Jennifer Fleming and Richard Koman. 1998. Web navigation: designing the user experience. O'Reilly, Sebastopol, CA, USA.

[14] Jane E Fountain. 2000. Constructing the information society: women, information technology, and design. Technology in Society 22 (2000), 45-62.

[15] Batya Friedman. 1996. Value-sensitive design. Interactions 3, 6 (1996), 16-23.

[16] Charles G Hill, Maren Haag, Alannah Oleson, Chris Mendez, Nicola Marsden, Anita Sarma, and Margaret Burnett. 2017. Gender-Inclusiveness Personas vs. Stereotyping: Can We Have it Both Ways?. In Proceedings of the 2017 CHI Conference on Human Factors in Computing Systems. ACM, 6658-6671.

[17] Eva Källhammer and Åsa Wikberg-Nilsson. 2012. Gendered innovative design: critical reflections stimulated by personas. Promoting innovation: Policies, Practices and Procedures (2012), 328-350.

[18] Christopher F Karpowitz, Tali Mendelberg, and Lee Shaker. 2012. Gender inequality in deliberative participation. American Political Science Review 106, 3 (2012), 533-547.

[19] Sam Killermann. 2017. A Guide to Gender: The Social fustice Advocate's Handbook. Impetus Books, Austin, TX.

[20] Milena Ribeiro Lopes and Carl Vogel. 2017. Women's Perspective on Using Tinder: A User Study of Gender Dynamics in a Mobile Device Application. In Proceedings of the 35th ACM International Conference on the Design of Communication (Halifax, Nova Scotia, Canada) (SIGDOC '17). ACM, New York, NY, USA, Article 12, 10 pages.

Figure 5: Ranks of women and men for task 2: data ordered by medians and means values. 
[21] Milena Ribeiro Lopes and Carl Vogel. 2019. Gender Differences in Online Dating Experiences. In It Happened on Tinder: Reflections and Studies on Internet-Infused Dating, Amir Hetsroni and Meriç Tuncez (Eds.). Institute of Network Cultures, Amsterdam, NL, 31-47.

[22] Milena Ribeiro Lopes and Carl Vogel. 2020. Gender Effects in Mobile Application Development. In Proceedings of the 1st IEEE International Conference on HumanMachine Systems. IEEE, New York, NY, USA, 1-6.

[23] Nicola Marsden and Maren Haag. 2016. Stereotypes and politics: reflections on personas. In Proceedings of the 2016 CHI Conference on Human Factors in Computing Systems. ACM, 4017-4031.

[24] Adrienne L. Massanari. 2010. Designing for imaginary friends: information architecture, personas and the politics of user-centered design. New Media \& Society 12, 3 (2010), 401-416

[25] Steve Mulder and Ziv Yaar. 2006. The user is always right: A practical guide to creating and using personas for the web. New Riders Publishing, Thousand Oaks, CA, USA.

[26] Nelly Oudshoorn, Els Rommes, and Marcelle Stienstra. 2004. Configuring the user as everybody: Gender and design cultures in information and communication technologies. Science, Technology, \& Human Values 29, 1 (2004), 30-63.

[27] John Pruitt and Tamara Adlin. 2006. The persona lifecycle: keeping people in mind throughout product design. Morgan Kaufmann, San Francisco, CA, USA.

[28] Els Rommes. 2006. Gender Senititve Design Practices. In Encyclopedia of gender and information technology, Eileen Moore Trauth (Ed.). IGI Global, Hershey, PA, USA, 675-681.
[29] Els Rommes. 2014. Feminist interventions in the design process. In Gender in Science and Technology. Transcript Verlag, Bielefeld, DE, 41-55.

[30] Els Rommes, Corinna Bath, and Susanne Maass. 2012. Methods for intervention: Gender analysis and feminist design of ICT. Science, Technology, \& Human Values 37, 6 (2012), 653-662.

[31] Phoebe Sengers, Kirsten Boehner, Shay David, and Joseph 'Jofish' Kaye. 2005. Reflective Design. In Proceedings of the 4th Decennial Conference on Critical Computing: Between Sense and Sensibility (Aarhus, Denmark) (CC '05). ACM, New York, NY, USA, 49-58.

[32] Phil Turner and Susan Turner. 2011. Is stereotyping inevitable when designing with personas? Design studies 32, 1 (2011), 30-44.

[33] Lisa Wade. 2017. American Hookup: The new culture of sex on campus. WW Norton \& Company, New York, NY, USA.

[34] Judy Wajcman. 1991. Feminism confronts technology. Polity Press, Cambridge, UK

[35] Judy Wajcman. 2000. Reflections on Gender and Technology. Social Studies of Science 30, 3 (2000), 447-464.

[36] Å. Wikberg-Nilsson, E. Källhammer, Y. Fältholm, and L. Abrahamsson. 2010. Personas as a method for applying gender theory in Triple Helix constellations: experiences from two research projects. In Proceedings of the VIII Triple Helix International Conference on University, Industry and Government Linkages. Triple Helix Association, W29:1-W29:13.

[37] Gayna Williams. 2014. Are you sure your software is gender-neutral? Interactions 21,1 (2014), 36-39. 